\title{
Cancer Mutations and their Tissue-specific Nature Blighe $\mathrm{K}^{*}$
}

Fondazione IRCCS Ca' Granda - Ospedale Maggiore Policlinico, Palazzina Bertarelli, Italy

\section{Introduction}

There are certain genes that have ubiquitous expression patterns in an individual's cells; yet, a paradox exists whereby mutations (germline or somatic) in such genes are only strongly associated with cancers of specific tissues. As these genes are ubiquitously expressed in the body's cells and thereby have functions in -potentially- all tissue types, then surely their resulting defects would manifest as cancers in all tissues?

If we take a snapshot of these genes and explore their functions and the circumstances in which they are required in a cell, the apparent 'tissue-specific' paradox becomes less of a paradox and more comprehensible, on a fundamental level. Examples of these include (but are not limited to) ATM, BRCA1, BRCA2, BRIP1, ERCC3, ERCC4, ERCC5, FANCA, FANCD2, FANCG, MLH1, MSH2, MUTYH, PMS1, PMS2, RECQL4, WRN, XPA, and XPC. As we will see later in this commentary, these genes are most commonly associated with cancers of the breast, skin, and colon; moreover, we find that this subset of genes is mostly enriched ( $p<0.0001$ with Bonferroni correction) for the Gene Ontology (GO) [1] terms 'DNA repair' (GO:0006281), 'response to DNA damage stimulus' (GO:0006974), 'DNA metabolic process' (GO:0006259), and 'cellular response to stress' (GO:0033554).

Thus, it can be inferred that the answer to the question posed in the opening paragraph of this commentary can be found by focusing on DNA repair and how a cell copes with stressors. Indeed, it will be seen later that it is the type of DNA damage that is induced in a tissue's cells that is key: double-strand breaks feature mostly in the breast and ovary; UV radiation-induced damage in the skin; and basemismatch in the colon. This corroborates with the long-held notion that endogenous and exogenous stress components have a role to play in cancer predisposition [2,3], but this has been somewhat ignored in cancer research, with groups tending to instead focus more on individual cancers as opposed to all cancers in general, with one recent and noteworthy exception [4].

Thus, in order to completely understand this 'tissue-specific' paradox some more, we have to consider why DNA damage is different in these parts of the body. First, however, we should try to understand which the genes are and what their roles are when DNA becomes damaged. As a detailed example, let's look at ionizing radiation, which induces DNA strand breaks [5].

\section{Breast Cancer and DNA Double-strand Breaks}

Taking breast tissue and cancer as a detailed example, we can understand why DNA damage has a role to play in the apparent tissue-specific nature of these genes in increasing cancer risk. From Reinhardt and Schumacher [6], we can build a simplistic model of the process (Figure 1). In this model, ATM is activated once DNA damage has occurred. In turn, TP53 is activated, as are MDM2 and WIP1. TP53 then activates BRCA1 and members of the Fanconi Anemia complementation group of proteins (FANC $[\mathrm{X}]$ ), which includes BRCA2, in order to initiate DNA repair (double-strand break repair in the case of BRCA1). Concurrently, MDM2 and WIP1 act to downregulate TP53 and thus the DNA repair process; however, TP53 continues to become activated in 4-7 hour 'pulses' until the DNA damage has been repaired. This model for DNA repair has also been explored and discussed by Jackson [5].

From this DNA damage response (DDR) process, we can hypothesize certain possibilities: Firstly, non-functioning TP53 could result in DNA damage accumulation and possible neoplastic transformation. This would occur if TP53 failed to activate BRCA1 and $\mathrm{FANC}[\mathrm{X}]$ after damage was induced. With no way of sensing the damaged DNA, the cell(s) would continue to attempt to function and divide, leaving the possibility of further accumulation of damage and possibly resulting in neoplastic transformation. This possibility has been somewhat confirmed, already, as TP53 mutations can result in Li-Fraumeni Syndrome, which heightens risk for many cancers and results in tumor growths in different tissues [7].

Secondly, if we instead had non-functioning BRCA1 -and, thus, the lack of an actual mechanism to repair the damaged DNA- this could result in repeated activation of TP53 in the DDR. This could itself, plausibly, result in different outcomes: With sustained and heightened activity of TP53, the cell could be pushed past its Hayflick Limit through rapid and repeated division and, as a result, lead to the

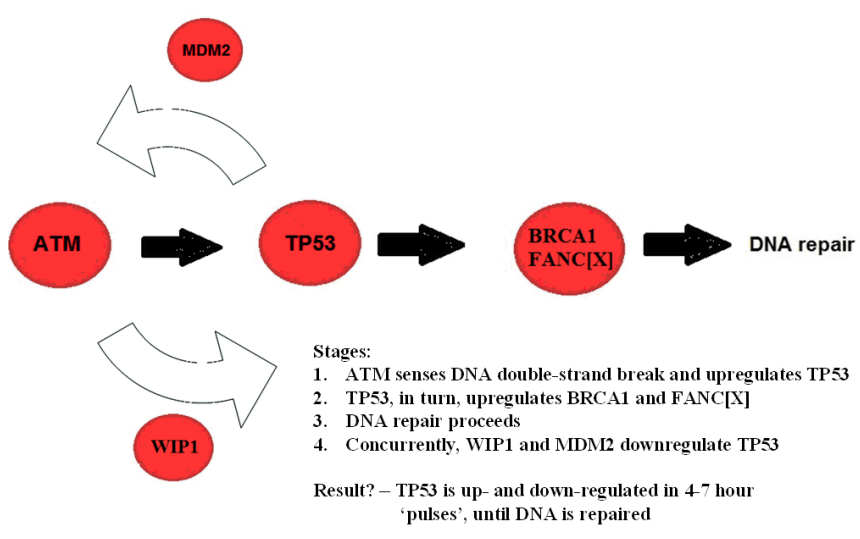

Figure 1: Simplistic model of DNA repair involving ATM, TP53, and the BRCA $1 / 2$ and FANC $[X]$ complexes. ATM and TP53 are master regulators in this process where signaling occurs in 'pulses' until the damaged DNA has been repaired. Failure of any one of these players could result in altered DNA repair mechanisms.

*Corresponding author: Blighe K, Angelo Bianchi Bonomi Hemophilia and Thrombosis Center, Fondazione IRCCS Ca' Granda - Ospedale Maggiore Policlinico, Fondazione Luigi Villa, 20122 Milan, Italy, Tel: +39 02 55035414; E-mail: kevin.blighe@policlinico.mi.it

Received August 27, 2013; Accepted January 21, 2014; Published January 24 2014

Citation: Blighe K (2014) Cancer Mutations and their Tissue-specific Nature. J Cancer Sci Ther 6: 009-011. doi:10.4172/1948-5956.1000241

Copyright: (c) 2014 Blighe K. This is an open-access article distributed under the terms of the Creative Commons Attribution License, which permits unrestricted use, distribution, and reproduction in any medium, provided the original author and source are credited. 
senescence and aging of the damaged genome $[6,8]$. In this case, the chance could also exist whereby the cell became neoplastic, as cells pushed past their Hayflick Limit have 'uncapped' telomeres, which are normally interpreted by the cellular machinery as double-strand breaks, thus leading back to the original problem when DNA damage was first induced. For this to occur, however, mutations in other key checkpoint genes -such as TP21 and/or TP16- would be required, in which case the cell would enter a pseudo-immortal state (explored in the proceeding paragraph). Alternatively, repeated activation of TP53 could result in the repeated apoptosis of the damaged cell, thus depleting the stem cell compartments; although, this outcome requires further probing.

Pertaining to our simple model (Figure 1), Robles and Adami [8] and Scully and Livingston [9] mention how, in the absence of BRCA1/2, TP21 is activated. TP21 is a TP53-dependent cell-cycle inhibitor and is thus part of a 'DNA-damage-dependent' checkpoint measure. The activation of TP21 prevents the death of the cell in which DNA damage has occurred; however, this cell-cycle delay can result in 'death by checkpoint' [8], whereby the cell can neither repair the DNA that's damaged nor continue to function viably. Interestingly, in Fanconi Anemia (associated with the FANC[X] complementation group, which are involved in DNA repair and interact with BRCA1), short stature and developmental disabilities are a feature - are these delayed and stunted growths due to repeated cell-cycle arrest related to TP21 as described above? In Fanconi Anemia, we also see the failure of hematologic components to develop, leading to a compromised immune and oxygen delivery system.

Thus, we have two possibilities in relation to BRCA1 deactivation:

- Deactivation in a genome that is otherwise healthy, which leads to cell-cycle arrest through TP21 in the DNA damaged cell ('death by checkpoint') and ceasing/arresting of cell-division.

- Deactivation in a genome that that also has certain checkpoint genes deactivated (for example, TP21), in which case the damaged DNA would be tolerated (or, better put: unrecognized) and the cell would continue to divide, possibly fuelling neoplastic transformation in a similar mode as already described.

\section{BRCA1 Screening Alone Cannot Confer 'True' Cancer Risk}

Thus, for cancer to occur in BRCA1 mutation carriers, mutations in other checkpoint genes must occur. This explains why not all carriers of germline mutations in this gene develop cancer, and why there's no syndrome associated with BRCA1 mutations. This also implies that screening for just BRCA1 mutations in, for example, familial -'highrisk'- cancer is not enough to deduce 'true' cancer risk; rather, mutations in other key checkpoint genes must also be screened in conjunction with those in BRCA1. These should be carried out as standard in clinical laboratories that are testing for BRCA1 gene mutations.

\section{BRCA1 and Tissue-specificity}

Now that we understand the role of BRCA1 in DNA repair -specifically double-strand breaks- we are better equipped to understand its tissue-specific nature in relation to breast cancer. DNA in breast tissue suffers damage from numerous sources, including ultraviolet (UV) and ionizing radiation, and also reactive oxygen species (ROS) [5]; whereas, other factors such as mechanical effects/stress $[10,11]$ and high breast tissue density [12] are important to consider in relation to cancer and risk too. Whilst UV radiation-induced DNA damage necessitates DNA repair through the nucleotide excision repair (NER) pathway, ionizing radiation, mechanical stress, and ROS can result in double-strand breaks, which require more complex repair mechanisms, such as homologous recombination (HR) [13].

The breast tissue responds to estrogen, specifically $17 \beta$-estradiol. When metabolized, this hormone produces ROS that can break the DNA double helix $[14,15]$. Thus, the reason why certain genes -specifically $A T M, B R C A 1, B R C A 2$, and BRIP1- are mostly associated with breast cancer is because of their role in repairing DNA doublestrand breaks, which occur mostly in the breast tissue as a result of sensitivity to estrogen. This additionally explains why these genes also increase risk for cancer of the ovaries, which are also estrogen-sensitive. It is worth noting again, at this point, that $B R C A 2$ and $B R I P 1$ are part of the Fanconi Anemia complementation group, a group from which 3 other members function in DNA double-strand break repair (FANCA, FANCD2, FANCG).

A question that remains in relation to this, however, is: why are BRCA1-related breast cancers typically triple-negative (estrogen-, progesterone-, and ERBB2-negative)? My hypothesis is based on the assumption that estrogen has a key involvement in DNA doublestrand breaks in breast and ovarian tissue through its metabolization to produce ROS. If any cells become neoplastic through this mechanism, they would have to be estrogen-sensitive to start. A possible explanation for this is that once damage has occurred, the cell is pushed through repeated cycles of division by TP53 (as mentioned previously), such that it gradually loses the original phenotype from which it derived, including receptor status. This could occur where TP53 remained activated but where the cell was unable to repair the damaged DNA. Also, progenitor stem cell populations could be decreased, thus the tissue would lose its 'stemness'.

\section{DNA Repair in Skin and Colon Cancer}

We can also extend our logic to understand why genes such as ERCC3, ERCC4, ERCC5, XPA, and XPC are mostly associated with skin cancer. Each of these genes is involved in DNA repair following UV light-induced damage through the NER pathway, and their deficiency can lead to xeroderma pigmentosum, which in itself usually leads to skin cancer. Research is mixed for their association to other cancers; for example, lung cancer and ERCC3 [16], breast cancer and ERCC4 [17], esophageal squamous cell carcinoma, gastric cancer, and ERCC5 $[18,19]$. Thus, it again appears that these genes are specific to skin cancer by their role in DNA repair via the NER pathway, which is activated after UV light-induced damage. It makes logical sense that the type of DNA damage most afflicting the skin would be from UV radiation from the Sun.

Interestingly, the genes that are mostly associated with colon cancer in the literature -i.e. MLH1, MSH2, MUTYH, PMS1, and PMS2are involved in an entirely different DNA repair pathway, being: DNA mismatch repair [20,21]. DNA mismatch occurs during standard DNA replication [22]; so, perhaps we see these genes associated with colon cancer more than cancers of the skin or breast because UV- and ionizing radiation are more common in these other parts of the body. As the colon is internal, it is somewhat shielded from external sources of radiation. Thus, it can be that DNA mismatch repair defects play key roles in many cancers, but that we see them mostly associated with colon cancer because it is the principle repair pathway required by this tissue. It could also be related to the rapid turnover of cells in the gut 
endothelium, which would result in a higher frequency of mismatched bases during replication. Diet must also be considered, and there are some reports of how this can have an effect on the gut endothelium $[23,24]$.

\section{Conclusion}

A key fact remains: Each of these genes is involved in repairing DNA, irrespective of the tissue in which DNA damage occurs. Thus, we can understand why each of these genes' defects/mutations can also be associated with cancers different from the one to which they are most strongly associated. For example, mutations in BRCA1 have also been associated to a lesser extent with pancreatic, uterine, and cervical cancer [25], whereas $M L H 1$ has been associated with breast cancer [26].

So, it is left to conclude that harboring mutations in a DNA repair gene broadly increases your risk of developing cancer. Risk will vary, however, from tissue to tissue, and this depends on the level and type of damage that occurs in each tissue and the subsequent DNA repair pathway that is then initiated.

\section{References}

1. Ashburner M, Ball CA, Blake JA, Botstein D, Butler H, et al. (2000) Gene ontology: tool for the unification of biology. The Gene Ontology Consortium. Nat Genet 25: 25-29.

2. Swenberg JA, Lu K, Moeller BC, Gao L, Upton PB, et al. (2011) Endogenous versus exogenous DNA adducts: their role in carcinogenesis, epidemiology, and risk assessment. Toxicol Sci 120: S130-145.

3. Trachootham D, Alexandre J, Huang P (2009) Targeting cancer cells by ROSmediated mechanisms: a radical therapeutic approach? Nat Rev Drug Discov 8: 579-591.

4. Alexandrov LB, Nik-Zainal S, Wedge DC, Aparicio SA, Behjati S, et al. (2013) Signatures of mutational processes in human cancer. Nature 500: 415-421.

5. Jackson SP (2002) Sensing and repairing DNA double-strand breaks. Carcinogenesis 23: 687-696.

6. Reinhardt HC, Schumacher B (2012) The p53 network: cellular and systemic DNA damage responses in aging and cancer. Trends Genet 28: 128-136.

7. Sorrell AD, Espenschied CR, Culver JO, Weitzel JN (2013) Tumor protein p53 (TP53) testing and Li-Fraumeni syndrome: current status of clinical applications and future directions. Mol Diagn Ther 17: 31-47.

8. Robles SJ, Adami GR (1998) Agents that cause DNA double strand breaks lead to $\mathrm{p} 16 \mathrm{INK} 4 \mathrm{a}$ enrichment and the premature senescence of normal fibroblasts. Oncogene 16: 1113-1123.

9. Scully R, Livingston DM (2000) In search of the tumour-suppressor functions of BRCA1 and BRCA2. Nature 408: 429-432.

10. Boyd NF, Martin LJ, Yaffe MJ, Minkin S (2011) Mammographic density and breast cancer risk: current understanding and future prospects. Breast Cancer Res 13: 223

11. Tse JM, Cheng G, Tyrrell JA, Wilcox-Adelman SA, Boucher Y, et al. (2012) Mechanical compression drives cancer cells toward invasive phenotype. Proc Natl Acad Sci U S A 109: 911-916.

12. Schreer I (2009) Dense Breast Tissue as an Important Risk Factor for Breast Cancer and Implications for Early Detection. Breast Care (Basel) 4: 89-92.

13. Khanna KK, Jackson SP (2001) DNA double-strand breaks: signaling, repair and the cancer connection. Nat Genet 27: 247-254.

14. Brown NS, Bicknell R (2001) Hypoxia and oxidative stress in breast cancer Oxidative stress: its effects on the growth, metastatic potential and response to therapy of breast cancer. Breast Cancer Res 3: 323-327.

15. Williamson LM, Lees-Miller SP (2011) Estrogen receptor a-mediated transcription induces cell cycle-dependent DNA double-strand breaks. Carcinogenesis 32: 279-285.

16. Hu Z, Xu L, Shao M, Yuan J, Wang Y, et al. (2006) Polymorphisms in the two helicases ERCC2/XPD and ERCC3/XPB of the transcription factor IIH complex and risk of lung cancer: a case-control analysis in a Chinese population. Cancer Epidemiol Biomarkers Prev 15: 1336-1340.

17. Milne RL, Ribas G, González-Neira A, Fagerholm R, Salas A, et al. (2006) ERCC4 associated with breast cancer risk: a two-stage case-control study using high-throughput genotyping. Cancer Res 66: 9420-9427.

18. Zhu ML, Shi TY, Hu HC, He J, Wang M, et al. (2012) Polymorphisms in the ERCC5 Gene and Risk of Esophageal Squamous Cell Carcinoma (ESCC) in Eastern Chinese Populations. PLoS One 7: e41500.

19. Duan Z, He C, Gong Y, Li P, Xu Q, et al. (2012) Promoter polymorphisms in DNA repair gene ERCC5 and susceptibility to gastric cancer in Chinese. Gene 511: 274-279.

20. Sinicrope FA, Foster NR, Thibodeau SN, Marsoni S, Monges G, et al. (2011) DNA Mismatch Repair Status and Colon Cancer Recurrence and Survival in Clinical Trials of 5-Fluorouracil-Based Adjuvant Therapy. J Natl Cancer Inst 103: 863-875.

21. Peltomäki $P$ (2001) Deficient DNA mismatch repair: a common etiologic factor for colon cancer. Hum Mol Genet 10: 735-740.

22. Kunkel TA, Erie DA (2005) DNA mismatch repair. Annu Rev Biochem 74: 681710.

23. Bartsch H, Nair J, Owen RW (2002) Exocyclic DNA adducts as oxidative stress markers in colon carcinogenesis: potential role of lipid peroxidation, dietary fat and antioxidants. Biol Chem 383: 915-921.

24. Bartsch H, Nair J (2002) Potential role of lipid peroxidation derived DNA damage in human colon carcinogenesis: studies on exocyclic base adducts as stable oxidative stress markers. Cancer Detect Prev 26: 308-312.

25. Thompson D, Easton DF; Breast Cancer Linkage Consortium (2002) Cancer Incidence in BRCA1 mutation carriers. J Natl Cancer Inst 94: 1358-1365.

26. Bianchi F, Raponi M, Piva F, Viel A, Bearzi I, et al. (2011) An intronic mutation in MLH1 associated with familial colon and breast cancer. Fam Cancer 10: 27-35. 